\title{
Cloud tolerance of remote-sensing technologies to measure land surface temperature
}

\author{
Thomas R. H. Holmes ${ }^{1,2}$, Christopher R. Hain ${ }^{3}$, Martha C. Anderson ${ }^{1}$, and Wade T. Crow ${ }^{1}$ \\ ${ }^{1}$ Hydrology and Remote Sensing Lab., USDA-ARS, Beltsville, MD, USA \\ ${ }^{2}$ Hydrological Sciences Laboratory, NASA Goddard Space Flight Center, Greenbelt, MD, USA \\ ${ }^{3}$ Earth Science Interdisciplinary Center, University of Maryland, College Park, MD, USA \\ Correspondence to: Thomas R. H. Holmes (thomas.r.holmes@nasa.gov)
}

Received: 13 April 2016 - Published in Hydrol. Earth Syst. Sci. Discuss.: 21 April 2016

Revised: 1 July 2016 - Accepted: 21 July 2016 - Published: 11 August 2016

\begin{abstract}
Conventional methods to estimate land surface temperature (LST) from space rely on the thermal infrared (TIR) spectral window and is limited to cloud-free scenes. To also provide LST estimates during periods with clouds, a new method was developed to estimate LST based on passivemicrowave (MW) observations. The MW-LST product is informed by six polar-orbiting satellites to create a global record with up to eight observations per day for each $0.25^{\circ}$ resolution grid box. For days with sufficient observations, a continuous diurnal temperature cycle (DTC) was fitted. The main characteristics of the DTC were scaled to match those of a geostationary TIR-LST product.

This paper tests the cloud tolerance of the MW-LST product. In particular, we demonstrate its stable performance with respect to flux tower observation sites (four in Europe and nine in the United States), over a range of cloudiness conditions up to heavily overcast skies. The results show that TIRbased LST has slightly better performance than MW-LST for clear-sky observations but suffers an increasing negative bias as cloud cover increases. This negative bias is caused by incomplete masking of cloud-covered areas within the TIR scene that affects many applications of TIR-LST. In contrast, for MW-LST we find no direct impact of clouds on its accuracy and bias. MW-LST can therefore be used to improve TIR cloud screening. Moreover, the ability to provide LST estimates for cloud-covered surfaces can help expand current clear-sky-only satellite retrieval products to all-weather applications.
\end{abstract}

\section{Introduction}

Information about the land surface temperature (LST) is an important element in the retrieval of many hydrological states and fluxes from satellite-measured radiances. For example, the retrieval of soil moisture or precipitation from passivemicrowave observations requires a coincident estimate of LST (e.g., Owe et al., 2008). In other applications, the rate of change in temperature is contrasted with net radiation to estimate evaporation as a residual of the surface energy balance (e.g., Anderson et al., 2011).

The most direct way to estimate LST from spaceborne instruments is by radiometers which measure within the thermal infrared (TIR) band of the electromagnetic spectrum. Thermal emission within this frequency band can be related directly to the physical temperature of the land surface and is more precisely termed the ensemble radiometric temperature (Norman and Becker, 1995). Spaceborne TIR radiometers allow for very high spatial resolution imagery. Even at the height of geostationary platforms radiometers can deliver $3 \mathrm{~km}$ spatial resolution, e.g., the Spinning Enhanced Visible and Infrared Imager (SEVIRI). A drawback to the TIR technique is that - at such wavelengths - clouds completely block the emission from the land surface. This means that spaceborne TIR radiometers give no information about the land surface below the clouds and instead reflect the temperature and emissivity of the clouds. The result is that the quality of the cloud screening directly affects the quality of a TIR-LST product.

An alternative, more cloud-tolerant technique is based instead on passive-microwave (MW) observations. In partic- 
ular the Ka-band $(\sim 37 \mathrm{GHz})$ was shown to have a strong link with LST (Owe and Van de Griend, 2001). Based on these observations, linear-regression-based LST estimates were derived for the Ka-band (Holmes et al., 2009), and variants of these linear relations are currently used in soil moisture retrieval (Jackson et al., 1999; Owe et al., 2008). However, using a single linear regression across the globe ignores potentially significant differences in microwave emissivity and can result in large biases, especially in desert areas. It also cannot account for large differences in the amplitude of the diurnal cycle between MW- and TIR-based LST, which have been implicated in reduced soil moisture retrieval skill during daylight hours (Lei et al., 2015; Parinussa et al., 2011). In contrast to these relatively simple linear methods, a neural network method was developed by Aires et al. (2001) to estimate LST based on multiple microwave channels besides Ka-band. By using atmospheric and surface information in addition to TIR-LST in the training of the scheme, this method minimized systematic bias in monthly mean temperatures. However, because the training is based on a single polar-orbiting satellite, it cannot give diurnal temperature information. Both of these methods were compared with station data by Catherinot et al. (2011), giving strong confirmation on the lack of sensitivity of microwave-based LST estimates to cloud liquid water path. This method has recently been developed further to allow for more continuous application to a more diverse suite of satellites and overpass times (Prigent et al., 2016). One drawback to all passivemicrowave-based methods is relatively coarse spatial resolution as compared to TIR sensors. At Ka-band, the smallest footprint size currently achieved with polar-orbiting satellites is $10 \times 15 \mathrm{~km}$.

Because of the complementary nature of TIR- and MWbased LST, there is a clear interest in merging these two independent technologies. For example, TIR-LST-based evaporation retrievals would benefit from observational data during cloudy periods (e.g., Anderson et al., 2011). On the other hand, microwave soil moisture retrievals from Soil Moisture Active Passive (SMAP) have the goal of $9 \mathrm{~km}$ spatial resolution, and this poses a resolution challenge to MW-LST inputs if TIR-LST cannot be leveraged. Reconciling the systematic differences in diurnal temperature cycle (DTC) between TIR and Ka-band is the first step towards an ultimate merger into a diurnally continuous LST product. To do this, Holmes et al. (2015) developed a method to scale the diurnal characteristics of a multi-satellite dataset of Ka-band observations to a TIR-LST product with 15 min temporal sampling from geostationary satellites. This scaling was able to account for biases in characteristics of the DTC related to Ka-band emissivity, sensing depth and atmospheric effects (Holmes et al., 2015). By explicitly taking account of systematic differences in DTC between TIR and Ka-band, this method is able to estimate LST at any time of day from sparse Ka-band observations. Note that a similar pixel-by-pixel approach was applied by André et al. (2015) over the Arctic region where a single satellite can provide diurnal sampling.

The aim of this paper is to evaluate the new global MWLST dataset in comparison with existing TIR-LST data over clear-sky days and particularly to test the assumption that MW-LST is tolerant to high levels of cloud coverage. Ground observations provide a common benchmark to test the relative accuracy of the two satellite products. Because the diurnal MW-LST product (Holmes et al., 2015) was scaled to TIR-based LST as produced by the Land Surface Analysis Satellite Application Facility (LSA-SAF; see http://landsaf. meteo.pt), the evaluation is mostly concerned with temporal precision, not with absolute bias. In previous work it was shown that relative aspects of a coarse-scale product can be evaluated using sparse in situ observations (Holmes et al., 2012). For a thorough discussion of absolute accuracy, readers are referred to papers detailing validation exercises for LSA-SAF-LST (e.g., Ermida et al., 2014; Göttsche et al., 2013, 2016).

After establishing the accuracy of MW-LST relative to TIR-LST for a particular site, the stability of the precision of MW-LST (relative to ground data) for increasing cloudiness will be tested. Previous work showed indications of cloud tolerance of MW-LST in comparison to TIR-LST (Holmes et al., 2015), but the analysis used proxies for both cloud cover and LST quality. In this paper we use a more direct estimate of cloudiness and provide a more detailed look at the validation statistics for different levels of daytime cloudiness with the ground station as the reference. The hypothesis we test is that clouds affect a satellite-measured LST by introducing an error $(E)$. If $E$ is consistent in sign throughout the measurement period (e.g., if clouds always lower the satellite LST estimate), this will introduce a systematic bias that will increase with cloud cover. If on the other hand the sign of $E$ varies, it will increase the random error in LST, but not necessarily a systematic bias. Only if we do not see a systematic bias with increasing cloud cover, nor an increase in random error, can we reject the hypothesis that clouds affect the satellite LST.

\section{Materials}

\subsection{Satellite LST estimates: thermal infrared}

TIR-LST is available from many sources, including both low Earth-orbiting satellites and geostationary satellites. Because of our interest in the diurnal features of LST, used in surface energy balance evaluations, this study focuses on TIRLST products developed from geostationary satellites. The first product is based on the SEVIRI aboard the Meteosat Second Generation (MSG-9) satellite. MSG-9 is positioned over the Equator at $0^{\circ}$ longitude. It has geographic coverage of Africa, Europe and the east coast of South America (with incidence angles below $70^{\circ}$ ). The Land Surface Anal- 
ysis, Satellite Application Facility (LSA-SAF) produces operational LST products based on split-window observations (channels centered at 10.8 and $12.0 \mu \mathrm{m}$ ) of MSG-9. LSASAF-LST is originally produced at $3 \mathrm{~km}$ spatial resolution. For this study, the data are aggregated to match the $0.25^{\circ}$ resolution of MW-LST. If two-thirds of the $3 \mathrm{~km}$ observations are masked, then the sample average is rejected for that location and time.

For North America, NOAA operates the Geostationary Operational Environmental Satellites (GOES). GOES Surface and Insulation Products (GSIP) are produced by the Office of Satellite Data Processing and Distribution (OSPO), National Environmental Satellite Data and Information Service (NESDIS), NOAA, and include LST (V3 was used for this study). Unlike MSG-based LST, GOES GSIP LST is based on a dual-window technique (3.9 and $11.0 \mu \mathrm{m})$ rather than the preferred split-window technique due to the lack of a $12 \mu$ thermal channel on the current generation of the GOES imager. An operational hourly LST at $0.125^{\circ}$ spatial resolution is available from 2 April 2009 onwards. For this study, we averaged the nine $0.125^{\circ}$ nodes that cover the edge and center of the $0.25^{\circ} \mathrm{MW}$-LST product grid cell. In order to further reduce possible cloud contamination, a particular data point is only used if all nine $0.125^{\circ}$ pixels covering the $0.25^{\circ}$ grid box contain (non-cloud-masked) data.

\subsection{Satellite LST estimates: microwave}

The MW-LST product is based on vertical polarized Ka-band (36-37 GHz) brightness temperature $\left(T^{\mathrm{Ka}}\right)$ as measured by microwave radiometers on six satellites in low Earth orbit. These satellites include the Advanced Microwave Scanning Radiometer on EOS (AMSR-E) to October 2011 and its successor on AMSR2 from July 2012. Also included are the Special Sensor Microwave and Imager (SSM/I) on platforms F13, F14 and F15 of the Defense Meteorological Satellite Program; the Tropical Rainfall Measurement Mission (TRMM) Microwave Imager (TMI); and Coriolis-WindSat. These observations are combined to create a global record with up to eight observations per day for each $0.25^{\circ}$ resolution grid box. The data are binned in $15 \mathrm{~min}$ windows of local solar time (00:00-00:15 is the first window of the day). The brightness temperatures are intercalibrated using observations from the TRMM satellite (with an equatorial overpass) as a transfer reference. Individual $0.25^{\circ}$ averages are masked if the spatial standard deviation of the oversampled Ka-band observations exceeds a prior determined threshold for a given grid box. Both the intercalibration and quality control procedures are described in detail in Holmes et al. (2013a).

The methodology to estimate LST from this record of Kaband observations is described in Holmes et al. (2015) and summarized below. For days with suitable observations (a minimum of four, including at least one within a third day length from solar noon) and no $T^{\mathrm{Ka}}<250 \mathrm{~K}$ (an indication of frozen soil), a continuous DTC is fitted. The DTC model used is based on Göttsche and Olesen (2001) with slight adaptations to limit the number of parameters. This implementation (DTC3) is fully described in Holmes et al. (2015). DTC3 summarizes the DTC with two daily parameters (daily minimum $T_{0}$ at the start and end of the day, and diurnal amplitude $A$ ) together with diurnal timing $(\varphi)$, which is assumed a temporal constant (Holmes et al., 2013b). The daily mean is defined by the daily minimum and the amplitude $\left(\bar{T}=T_{0}+A / 2\right)$. The Ka-band DTC parameters for individual days $\left(\bar{T}_{d}^{\mathrm{Ka}}, A_{d}^{\mathrm{Ka}}\right)$ are scaled to match the long-term mean of TIR observations:

$A_{d}^{\mathrm{MW}}=A_{d}^{\mathrm{Ka}} / \delta$,

$\bar{T}_{d}^{\mathrm{MW}}=\beta_{0}+\beta_{1} \bar{T}_{d}^{\mathrm{Ka}}$.

The scaled parameters are indicated with the superscript "MW". The parameter $\delta$ represents the slope of the zeroorder least-squares regression line for estimating the amplitude of $A_{d}^{\mathrm{Ka}}$ from TIR-LST $\left(A_{d}^{\mathrm{TIR}}\right)$. The intercept $\left(\beta_{0}\right)$ and slope $\left(\beta_{1}\right)$ to correct the mean daily temperature $\left(\bar{T}_{d}^{\mathrm{Ka}}\right)$ for systematic differences with TIR-LST $\left(\bar{T}_{d}^{\mathrm{TIR}}\right)$ are determined with a constrained numerical solver, as in Holmes et al. (2015). The constraint is based on radiative transfer considerations and assures that the scaling of the mean is in agreement with the prior scaling of the amplitude (Eq. 1). These scaling parameters were determined for each $0.25^{\circ}$ grid box based on data for the period 2009-2012. The scaling (Eqs. 1 and 2) is applied to every day for which estimates of $\bar{T}^{\mathrm{Ka}}$ and $A^{\mathrm{Ka}}$ are available. Together with the timing of the diurnal cycle of TIR-LST, $\varphi^{\text {TIR }}$, as determined based on (Holmes et al., 2013b), we then calculate the diurnal MWLST based on the same DTC3 model:

MW-LST $=$ DTC3 $\left(\varphi^{\mathrm{TIR}} T_{0}^{\mathrm{MW}} A^{\mathrm{MW}}\right)$.

Comparing actual Ka-band observations to estimates provided by the fitted DTC model provides a valuable means of quality control. The root mean square error (RMSE) of the misfit between the DTC 3 model and the sparse $T^{\mathrm{Ka}}$ observations is used to flag days where the assumptions imposed by the shape of clear-sky DTC are not valid or individual Kaband observations have a large bias.

Besides the continuous MW-LST product, we can also evaluate the product at the actual Ka-band observation times (thus weakening our reliance on the DTC3 model). To do this, we project the difference between the original MW data and the DTC model fit onto MW-LST. This product is referred to as MW-LST-Sparse:

$$
\begin{aligned}
& \text { MW-LST-Sparse }=\operatorname{DTC} 3\left(\varphi^{\mathrm{TIR}}, T_{0}^{\mathrm{MW}}, A^{\mathrm{MW}}\right) \\
& +\frac{1}{\delta}\left(T^{\mathrm{Ka}}-\operatorname{DTC} 3\left(\varphi^{\mathrm{Ka}}, T_{0}^{\mathrm{Ka}}, A^{\mathrm{Ka}}\right)\right) .
\end{aligned}
$$

In MW-LST-Sparse the impact of the DTC 3 model is limited to providing the minimum and amplitude of the diurnal. The 
differences between the observations and the diurnal model at the actual observation time are preserved. The difference between the continuous MW-LST and the MW-LST-Sparse is illustrated in Fig. 1 (lower panel).

\subsection{Ground observations}

FLUXNET is a worldwide network of meteorological measurement towers (flux tower) with common measurement protocols (Baldocchi et al., 2001). Each flux tower includes an instrument positioned above the vegetation canopy to measure net radiation. This instrument is made up of two pyranometers to measure up- and downwelling shortwave radiation and two pyrgeometers to measure up- and downwelling longwave radiation. The radiometric surface temperature $(T)$ can be derived from the longwave radiation measurements (upwelling: $L^{\uparrow}, \mathrm{W} \mathrm{m}^{-2}$; downwelling: $L^{\downarrow}$, $\mathrm{W} \mathrm{m}^{-2}$ ) using the following equation:

$L^{\uparrow}=\varepsilon_{L} \sigma T^{4}+\left(1-\varepsilon_{L}\right) L^{\downarrow}$,

where $\varepsilon_{L}$ is the broadband emissivity over the spectral range of the pyrgeometer $(4.5-42 \mu \mathrm{m})$ and $\sigma$ is the StefanBoltzmann constant $\left(\sigma=5.670 \times 10^{-8} \mathrm{~W} \mathrm{~m}^{-2} \mathrm{~K}^{-1}\right)$.

\subsubsection{Longwave emissivity}

An estimate of $\varepsilon_{L}$ is obtained for each site based on measurements of $L^{\uparrow}$ together with additional measurements of screen level air temperature $\left(T_{\mathrm{a}}\right)$, sensible heat flux $(H)$ and wind speed $(u)$. This estimate is based on three assumptions: (1) $H$ is directly proportional to the near-surface temperature gradient, (2) the difference $T-T_{\mathrm{a}}$ represents that temperature gradient and (3) $H=0$ when there is no vertical temperature gradient (i.e., $T=T_{\mathrm{a}}$ ). With these three assumptions in mind we then iterate over $\varepsilon_{L}$ to find the solution where the regression of $H$ against $\left(T-T_{\mathrm{a}}\right.$ ) goes through zero and the squared errors are minimized. When measurements of wind speed are available, they are used to select atmospheric conditions where the relationship between $H$ and the near-surface temperature gradient is strongest $\left(u>2 \mathrm{~m} \mathrm{~s}^{-1}\right)$. For forest sites, the direct relationship between $H$ and $T$ gradients breaks down. In those cases, the simpler assumption is used that the long-term average of $T$ and $T_{\mathrm{a}}$ are equal: $\langle T\rangle=\left\langle T_{\mathrm{a}}\right\rangle$. For more discussion and examples of this method see Holmes et al. (2009). In this study we apply this method to determine monthly $\varepsilon_{L}$ for each site individually and then use the median value of $\varepsilon_{L}$ (listed in Table 1) to calculate $T$ based on Eq. (5). The standard deviation of the monthly measurements of $\varepsilon_{L}$ is also listed in Table 1 and provides an indication of both uncertainty and seasonal variation in $\varepsilon_{L}$.

\subsubsection{Spatial representativeness and site selection}

The tower-based estimate of $T$, from (Eq. 5), directly represents only the immediate tower surroundings within a ra- dius of approximately $50 \mathrm{~m}$. Clearly this is a very small spatial sampling of the $0.25^{\circ}$ grid box $(\sim 25 \times 25 \mathrm{~km})$ represented by the satellite LST estimates used in this study (see Sects. 2.1 and 2.2). As a consequence, we typically find large systemic differences between the station data and the areal average. Given that overall weather conditions are relatively homogenous over distances of $25 \mathrm{~km}$, these differences can be attributed to the land cover type at the station location in comparison to that over the entire grid box (for examples of this, see Holmes et al., 2009). The representation of the spatial average by ground observations can be improved significantly if more than one station is available in the same grid box and the towers are situated in thermodynamically contrasting land cover types (forest and cropland/herbaceous). In that case the land cover associated with the tower site (subscript, s) determines the weight $(W)$ according to the spatial fraction of that land cover type within the $0.25^{\circ}$ grid box (MCD12C1; Friedl et al., 2010). We use this information to estimate the grid average LST as the weighted average of site-measured $T$ according to Eq. (6):

$\mathrm{LST}=\frac{\sum_{s=1}^{n} W_{\mathrm{s}} T_{\mathrm{s}}}{\sum_{s=1}^{n} W_{\mathrm{s}}}$.

For example, site DE-Hai of location A is located in a forest and represents $16 \%$ of the pixel. Site DE-Geb is located in croplands and represents $80 \%$ of the pixel that has low vegetation cover or bare soil. Urban, open water or wetland accounts for the remaining $4 \%$, which does not affect the weighting. We only considered locations where this rest fraction is below $5 \%$ of the grid coverage. Another criterion for site selection was that the land cover at the site must represent more than $75 \%$ of the pixel. Sites in mountainous areas are also excluded. For the period of 2009-2012 this means there are 13 locations with at least 2 years of flux tower sites available for this study, and four of these locations contain multiple stations. For pixels where only one station is available, LST is set equal to the site measurement: $\mathrm{LST}=T$. All the validation targets are listed in Table 1, together with the geographic location of the individual stations, the land cover type as reported by the flux tower operators and the parameters $W$ and $\varepsilon_{L}$ as described above.

\subsubsection{Cloudiness at tower location}

The downwelling shortwave radiation $\left(S^{\downarrow}\right)$ as measured at the flux tower is strongly affected by the amount of condensed water in the atmosphere. We can therefore use the reduction in site-measured daytime $S^{\downarrow}$ relative to an expected value during clear skies as a proxy for cloudiness. The clearsky irradiance $S_{\text {clear }}^{\downarrow}$ is estimated based on top-of-atmosphere solar irradiance $\left(S^{\mathrm{TOA}}\right)$, which can be calculated based on geographic location and day of year (Van Wijk and Ubing, 

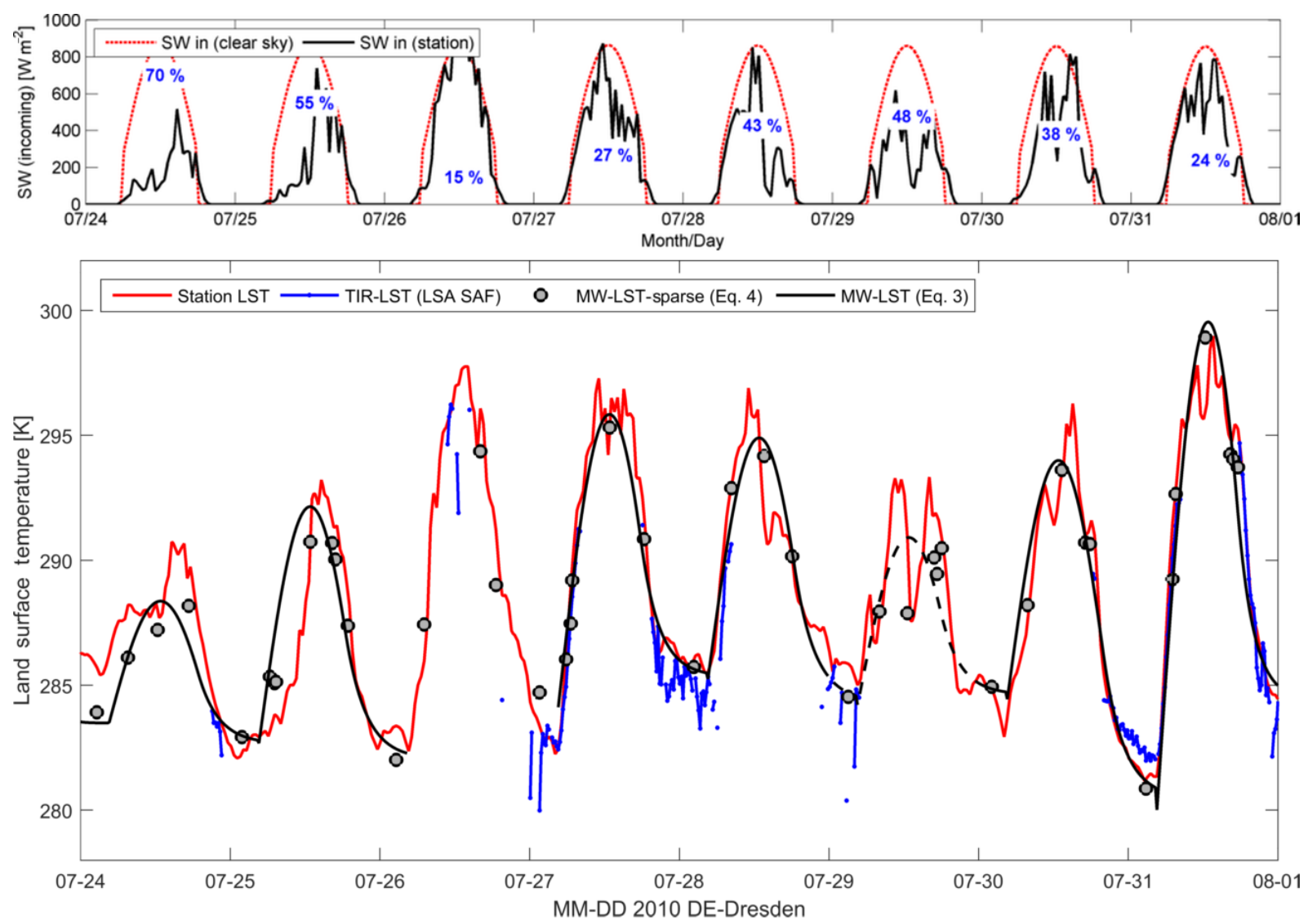

Figure 1. An example of the available data at station B, showing 8-day time series of station-measured shortwave incoming radiation ( $S^{\downarrow}$, or SW, top) and LST (bottom graph). In the top graph $S^{\downarrow}$ (black lines) is compared to the clear-sky expected value, $S_{\text {clear }}^{\downarrow}$ (Eq. 7, red dash), to illustrate the computation of the cloud cover proxy ( $A_{\text {cloud }}$, Eq. 8, values in blue text). In the lower graph the station LST is compared to the TIR and MW satellite LST products. The dashed line is an occurrence where the MW-LST is masked due to a high misfit between sparse observations and the diurnal model.

1963). Even on a clear day, atmospheric absorption reduces the irradiance at the surface by 20 to $30 \%$ from the topof-atmosphere value. We estimate this clear-sky absorption $\left(A_{\mathrm{clr}}\right)$ by calculating the slope $(\beta)$ of the zero-order linear regression between $S^{\downarrow}$ and $S^{\mathrm{TOA}}$ for days that are in the highest quintile of $S^{\downarrow} / S^{\mathrm{TOA}}: A_{\mathrm{clr}}=1-\beta$. These estimates of $A_{\mathrm{clr}}$ (listed in Table 1 for each individual site) range from 0.22 to 0.31 and show a good agreement between stations of the same cluster. We use the minimum recorded value for each cluster to calculate $S_{\text {clear }}^{\downarrow}$ :

$S_{\text {clear }}^{\downarrow}=S^{\mathrm{TOA}}\left(1-A_{\mathrm{clr}}\right)$.

By using $S_{\text {clear }}^{\downarrow}$ to normalize measured $S^{\downarrow}$, we account for solar zenith effects and can formulate a measure for shortwave cloud absorption $\left(A_{\text {cloud }}\right)$, expressed in percentage:

$A_{\text {cloud }}=100 \frac{S_{\text {clear }}^{\downarrow}-S^{\downarrow}}{S_{\text {clear }}^{\downarrow}}$.

This definition of $A_{\text {cloud }}$ is used as a measure of cloudiness and calculated based on $3 \mathrm{~h}$ totals of insolation for the day- time between 06:00 and 18:00. Obviously this definition of cloud absorption does not apply when the Sun is below the horizon. For nighttime hours we use the neighboring daytime window:

$$
\begin{aligned}
A_{\text {cloud }}(0-6) & =A_{\text {cloud }}(6-12), \\
A_{\text {cloud }}(18-24) & =A_{\text {cloud }}(12-18) .
\end{aligned}
$$

Figure 1 gives an example of the site-measured $S^{\downarrow}$ and the calculated $A_{\text {cloud }}$ for an 8-day summer period at station B (top panel). The bottom panel shows the site-measured LST and illustrates how the temporal sampling of the satellite products is affected by clouds.

\subsection{Statistical metrics}

In the description of the results we make use of standard statistical metrics. In terms of absolute error metrics we report bias, the long-term mean difference between satellite product and in situ data, and the RMSE. By removing the long-term mean difference, we can calculate RMSE of the unbiased 
Table 1. List of ground validation targets detailing geographic location and International Geosphere-Biosphere Programme (IGBP) vegetation type. Local parameters determined for each station are weight $(W)$ in spatial average, longwave emissivity and clear-sky absorption.

\begin{tabular}{|c|c|c|c|c|c|c|c|}
\hline Cluster ID, name & Site ID & $\begin{array}{l}\text { Geographic } \\
\text { location }\end{array}$ & Vegetation (IGBP) & $W$ & $\begin{array}{r}\varepsilon_{L} \\
\text { median } \\
(\mathrm{STD})\end{array}$ & $\begin{array}{r}\text { Clear-sky } \\
\text { absorbtion } \\
\left(A_{\mathrm{clr}}\right)\end{array}$ & Notes \\
\hline \multirow[t]{2}{*}{ A. DE-Thuringia } & DE-Hai & $\begin{array}{l}51.0792^{\circ} \mathrm{N} \\
10.453^{\circ} \mathrm{E}\end{array}$ & $\begin{array}{l}\text { Deciduous broadleaf } \\
\text { forest }\end{array}$ & .16 & $0.993(0.001)$ & 0.33 & * \\
\hline & DE-Geb & $\begin{array}{l}51.1001^{\circ} \mathrm{N} \\
10.9143^{\circ} \mathrm{E}\end{array}$ & Croplands & .80 & $0.983(0.004)$ & 0.31 & \\
\hline \multirow[t]{2}{*}{ B. DE-Dresden } & DE-Tha & $\begin{array}{l}50.9636^{\circ} \mathrm{N} \\
13.5669^{\circ} \mathrm{E}\end{array}$ & $\begin{array}{l}\text { Evergreen needleleaf } \\
\text { forest }\end{array}$ & .36 & $0.983(0.005)$ & 0.26 & \\
\hline & DE-Kli & $\begin{array}{l}50.8928^{\circ} \mathrm{N} \\
13.52250^{\circ} \mathrm{E}\end{array}$ & Croplands & .61 & $0.993(0.001)$ & 0.27 & \\
\hline \multirow[t]{2}{*}{ C. CZ-Billy Kris } & CZ-BK1 & $\begin{array}{l}49.50021^{\circ} \mathrm{N} \\
18.5368^{\circ} \mathrm{E}\end{array}$ & $\begin{array}{l}\text { Evergreen needleleaf } \\
\text { forest }\end{array}$ & .72 & $0.985(0.001)$ & 0.29 & * \\
\hline & $\mathrm{CZ}-\mathrm{BK} 2$ & $\begin{array}{l}49.4944^{\circ} \mathrm{N}, 1 \\
18.5429^{\circ} \mathrm{E}\end{array}$ & Grasslands & .23 & $0.985(0.001)$ & 0.30 & \\
\hline D. ES-LMa & ES-LMa & $\begin{array}{l}39.9415^{\circ} \mathrm{N} \\
5.7734^{\circ} \mathrm{W}\end{array}$ & Savannas & .77 & $0.987(0.001)$ & 0.25 & \\
\hline E. US-Marys River & US-MRf & $\begin{array}{l}44.6465^{\circ} \mathrm{N} \\
123.5515^{\circ} \mathrm{W}\end{array}$ & $\begin{array}{l}\text { Evergreen needleleaf } \\
\text { forest }\end{array}$ & 1.0 & $0.995(0.000)$ & 0.27 & \\
\hline F. US-Woodward & US-AR1 & $\begin{array}{l}36.4267^{\circ} \mathrm{N} \\
99.42^{\circ} \mathrm{W}\end{array}$ & Grasslands & .98 & $0.993(0.003)$ & 0.23 & \\
\hline G. US-SGP Main & US-ARM & $\begin{array}{l}36.6058^{\circ} \mathrm{N} \\
97.4888^{\circ} \mathrm{W}\end{array}$ & Croplands & 1.0 & $0.963(0.011)$ & 0.23 & \\
\hline H. US-Wind River & US-Wrc & $\begin{array}{l}45.8205^{\circ} \mathrm{N} \\
121.9519^{\circ} \mathrm{W}\end{array}$ & $\begin{array}{l}\text { Evergreen needleleaf } \\
\text { forest }\end{array}$ & .99 & $0.993(0.004)$ & 0.23 & \\
\hline \multirow[t]{2}{*}{ I. US-Santa Rita } & US-SRC & $\begin{array}{l}31.9083^{\circ} \mathrm{N} \\
110.8395^{\circ} \mathrm{W}\end{array}$ & Open shrublands & .5 & $0.960(0.008)$ & 0.21 & \\
\hline & US-SRM & $\begin{array}{l}31.8214^{\circ} \mathrm{N} \\
110.8661^{\circ} \mathrm{W}\end{array}$ & Woody savannas & .5 & $0.983(0.006)$ & 0.21 & \\
\hline J. US-Audubon & US-Aud & $\begin{array}{l}31.5907^{\circ} \mathrm{N} \\
110.5092^{\circ} \mathrm{W}\end{array}$ & Grasslands & .99 & $0.950(0.003)$ & 0.20 & \\
\hline K. US-Lucky Hills & US-Whs & $\begin{array}{l}31.7438^{\circ} \mathrm{N} \\
110.0522^{\circ} \mathrm{W}\end{array}$ & Open shrublands & 1.0 & $0.972(0.017)$ & 0.19 & \\
\hline L. US-Woodward & US-AR2 & $\begin{array}{l}36.6358^{\circ} \mathrm{N} \\
99.5975^{\circ} \mathrm{W}\end{array}$ & Grasslands & 1.0 & $0.992(0.003)$ & 0.26 & \\
\hline M. US-Kansas & US-KFS & $\begin{array}{l}39.0561^{\circ} \mathrm{N}, \\
95.1907^{\circ} \mathrm{W}\end{array}$ & Grasslands & 1.0 & $0.945(0.015)$ & 0.25 & \\
\hline
\end{tabular}

* Sites are located in neighboring grid cells. Reference: C: Marek et al. (2011).

data (ubRMSE). These three metrics are related as follows:

$\mathrm{RMSE}=\sqrt{\mathrm{ubRMSE}^{2}+\text { bias }^{2}}$.

We further report standard error of estimate (SEE) as a measure of temporal precision:

$\mathrm{SEE}=\sigma \sqrt{1-\rho^{2}}$, where $\sigma$ is the standard deviation of in situ data and $\rho$ is Pearson's correlation coefficient.

\section{Results}

We acquired data for 17 field sites in 13 unique grid locations with data records within the 4-year time period of 2009 to 
Table 2. Percentage coverage for two LST products.

\begin{tabular}{lrrrrr}
\hline & & \multicolumn{4}{c}{ MSG domain 2009-2011 } \\
\cline { 4 - 6 } & & \multicolumn{4}{c}{ Frost-free days } \\
\cline { 4 - 6 } \begin{tabular}{l} 
Sat \\
\cline { 4 - 6 } product
\end{tabular} & All & All & All & $\begin{array}{r}\text { Clear-sky } \\
\text { sky }\end{array}$ & $\begin{array}{r}\text { Cloudy } \\
\text { sky }\end{array}$ \\
\hline TIR & & & & 94 & 14 \\
MW & 56 & 42 & 47 & 75 & 56 \\
\hline
\end{tabular}

2012. Table 2 lists the amount of days with at least 12 hours of observations for either MW or TIR-LST to give an overall sense of available validation data for this study. In total we have 13316 data days of in situ data (36 out of 44 data years). Of these data days, $50 \%$ also have MW-LST estimates, and $36 \%$ have TIR-LST observations. For MW, this percentage is negatively affected by the gap between AMSR-E (radiometer turned off in October 2011) and AMSR2 (first observations in July 2012). The MW-LST product is heavily reliant on these satellites with a midday overpass for constraining the diurnal amplitude. For TIR, the percentage is particularly low for GOES due to a more stringent cloud filter than employed for MSG.

To better represent the relative data coverage that is possible with the two LST retrieval techniques, we focus on the four station pairs in the MSG domain and limit the time period to 2009-2011. We further focus on the days where the minimum temperature (as measured at the station) stays above freezing (the MW method is not applicable for subfreezing temperatures). Within this smaller subset, we have 2506 data days of in situ data, and the coverage of MW is $55 \%$ in comparison with the $42 \%$ coverage for TIR. However, breaking this down by cloud cover reveals the big difference in coverage resulting from the wavelength-dependent tolerance to clouds. During clear skies, the coverage of TIR is $93 \%$, and MW comes in at $76 \%$ (mainly attributed to the 2out-of-3-days revisit for ascending AMSR-E). During cloudy days the coverage drops to $13 \%$ for TIR, whereas for MW it maintains $55 \%$ coverage.

In the following section we want to answer two questions. How does the MW-LST compare to TIR-LST in relation to ground data during days with clear skies? And is the performance of MW-LST affected by clouds? We focus on hourly average temperatures for days where the station data remain above $1{ }^{\circ} \mathrm{C}$ to avoid snow or frozen surface conditions.

\subsection{Clear-sky comparison of satellite LST products}

The ground observations provide a common benchmark to test the relative accuracy and bias of the two satellite products with the same in situ data. Days with clear skies are selected based on the measure of cloudiness as defined in Sect. 2.3.3, with a maximum accepted value of $A_{\text {cloud }}=0.2$. This is in addition to the cloud screening performed in the
Table 3. Summary of "clear-sky" validation results.

\begin{tabular}{|c|c|c|c|c|}
\hline European FLUXNET & Statistic & TIR-LST & MW-LST & $\begin{array}{r}\text { MW-LST- } \\
\text { Sparse }\end{array}$ \\
\hline \multirow[t]{3}{*}{ A: DE-Thuringia } & ubRMSE & 2.2 & 2.4 & 2.4 \\
\hline & Bias & 0.4 & 1.2 & 1.1 \\
\hline & $\mathrm{N}$ & 2050 & 2636 & 525 \\
\hline \multirow[t]{3}{*}{ B: DE-Dresden } & ubRMSE & 1.6 & 1.7 & 1.7 \\
\hline & Bias & -0.6 & -0.7 & -0.9 \\
\hline & $\mathrm{N}$ & 4157 & 4521 & 1245 \\
\hline \multirow[t]{3}{*}{ C: CZ-BK } & ubRMSE & 2.6 & 2.6 & 2.6 \\
\hline & Bias & 2 & 2 & 1.9 \\
\hline & $\mathrm{N}$ & 2787 & 2985 & 850 \\
\hline \multirow{3}{*}{ D: ESLMa } & ubRMSE & 1.7 & 2.5 & 2.6 \\
\hline & Bias & 0.4 & 0.8 & 0.7 \\
\hline & $\mathrm{N}$ & 12055 & 9652 & 2560 \\
\hline \multirow{2}{*}{ Multi-site average } & ubRMSE & 2.1 & 2.3 & 2.3 \\
\hline & |Bias $\mid$ & 0.9 & 1.2 & 1.2 \\
\hline AmeriFlux & Statistic & TIR-LST & MW-LST & $\begin{array}{l}\text { MW-LST- } \\
\text { Snarse }\end{array}$ \\
\hline \multirow{3}{*}{ E: US-Marys River } & ubRMSE & 3.2 & 3 & 3.3 \\
\hline & Bias & 1.9 & 1.5 & 1.7 \\
\hline & $\mathrm{N}$ & 2810 & 1991 & 756 \\
\hline \multirow[t]{3}{*}{ F: US-Woodward } & ubRMSE & 3.1 & 3 & 2.8 \\
\hline & Bias & -0.6 & -0.5 & -0.5 \\
\hline & $\mathrm{N}$ & 2925 & 10386 & 3811 \\
\hline \multirow[t]{3}{*}{ G: US-SGP Main } & ubRMSE & 2.8 & 3.3 & 3 \\
\hline & Bias & 0.5 & 0.4 & 0.6 \\
\hline & $\mathrm{N}$ & 2895 & 8362 & 3105 \\
\hline \multirow[t]{3}{*}{ H: US-Wind River } & ubRMSE & 2.5 & 2.3 & 2.5 \\
\hline & Bias & -0.9 & -1.1 & -1.1 \\
\hline & $\mathrm{N}$ & 4219 & 6915 & 1687 \\
\hline \multirow[t]{3}{*}{ I: US-Santa Rita } & ubRMSE & 2.9 & 3.5 & 3.3 \\
\hline & Bias & -1.5 & -0.7 & -1.2 \\
\hline & $\mathrm{N}$ & 8908 & 13478 & 5312 \\
\hline \multirow[t]{3}{*}{ J: US-Audubon } & ubRMSE & 2.4 & 3.4 & 3.4 \\
\hline & Bias & -1.3 & -1.3 & -1.6 \\
\hline & $\mathrm{N}$ & 3301 & 8345 & 3078 \\
\hline \multirow[t]{3}{*}{ K: US-Lucky Hills } & ubRMSE & 3.1 & 3.5 & 3.5 \\
\hline & Bias & -0.7 & 0.3 & -0.1 \\
\hline & $\mathrm{N}$ & 7870 & 10786 & 4545 \\
\hline \multirow[t]{3}{*}{ L: US-AR2 } & ubRMSE & 3.2 & 2.6 & 2.4 \\
\hline & Bias & -0.5 & -0.6 & -0.6 \\
\hline & $\mathrm{N}$ & 2033 & 8766 & 3124 \\
\hline \multirow[t]{3}{*}{ M: US-Kansas } & ubRMSE & 2.3 & 2.1 & 2 \\
\hline & Bias & -0.2 & -0.1 & -0.4 \\
\hline & $\mathrm{N}$ & 1454 & 4788 & 1627 \\
\hline \multirow[t]{2}{*}{ Multi-site average } & ubRMSE & 2.8 & 3 & 2.9 \\
\hline & |Bias| & 0.9 & 0.7 & 0.9 \\
\hline
\end{tabular}

generation of the TIR products (Sect. 2.1), the quality control of the MW-LST (Sect. 2.2) and the selection of frost-free days. Even though the spatial representativeness and uncertainty in $\varepsilon_{L}$ may insert systematic errors in the estimation of 

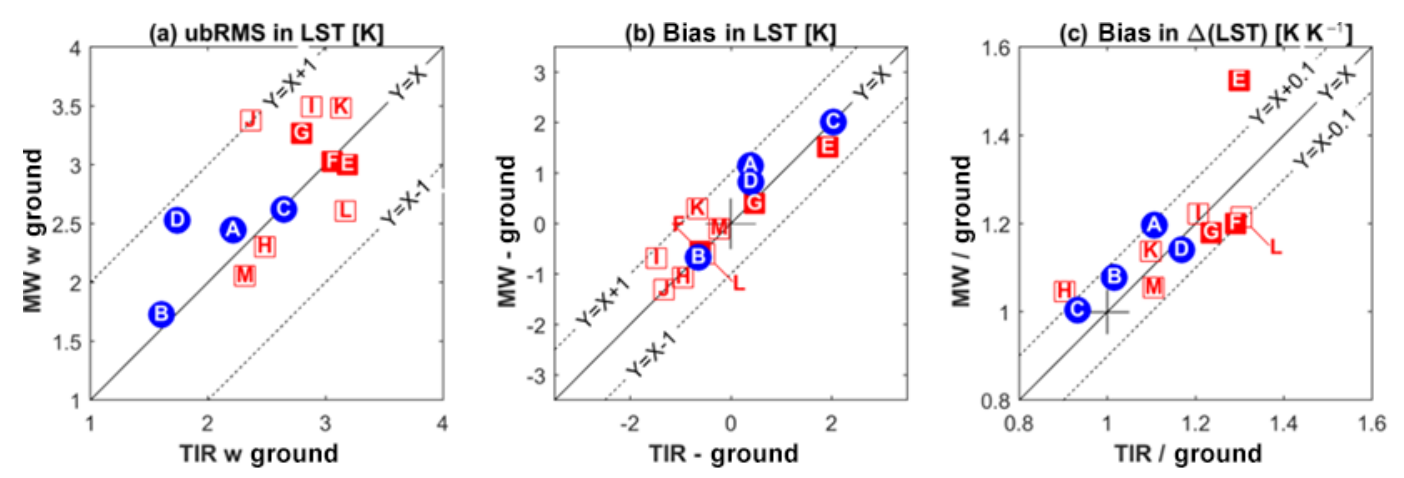

LST, MSG domain $\quad$ LST, GOES domain $\quad \square \quad$ LST, GOES domain

Figure 2. Comparison between TIR-LST ( $x$ axis) and MW-LST ( $y$ axis) in terms of their validation metrics with station LST for frost-free and cloud-free days. From left to right the three panels show (a) ubRMSE, (b) mean bias and (c) bias in $\Delta$ (LST). Each marker represents the statistics as calculated for individual locations as identified by the letter (see Table 1 for definition). For the GOES domain the filled markers highlight the stations used in the cloud analysis. Black lines provide visual support and indicate targets (e.g., 1 : 1 line, cross at zero bias).

the spatial average from the ground stations, they can be used as a reference to compare different satellite LST products.

For each of the 13 validation targets we tabulate (see Table 3) ubRMSE and bias (see Sect. 2.4 for definition). By excluding long-term bias, the ubRMSE gives an indication of the overall data quality, which includes the random error and errors resulting from a mismatch in variance (either seasonal or diurnal). Errors in spatial representation of the sites affect MW and TIR in the same way. In order to highlight the relative performance of the two satellite products with respect to the common benchmark, we compare their performance directly in Fig. 3.

Encouragingly, the multi-site average ubRMSE for the four European FLUXNET sites shows the MW-LST (2.3 K) to be only moderately higher than TIR-LST $(2.1 \mathrm{~K})$ for these frost-free and cloud-free observations. This is a positive result for MW-LST because of the extra processing needed to correct MW data for sensing depth differences with TIR. Both satellite products have a higher ubRMSE with the AmeriFlux stations, but again the multi-site average ubRMSE for MW-LST $(3.0 \mathrm{~K})$ is only slightly higher than that for TIR-LST $(2.8 \mathrm{~K})$. Figure 2a compares the ubRMSE with in situ data directly for the two satellite technologies. The high correlation between the two methods is an indication that the spurious effect of spatial representation of the site affects both methods to similar degrees. Of all the stations, MW-LST has a lower ubRMSE at 5 of the 13 stations, and the only stations where we record more than $0.5 \mathrm{~K}$ difference in ubRMSE between TIR and MW-LST are FLUXNET station D (2.5 K for MW vs. $1.7 \mathrm{~K}$ for TIR) and AmeriFlux stations I ( $3.5 \mathrm{~K}$ for MW vs. $2.9 \mathrm{~K}$ for TIR) and J (3.4 K for MW vs. $2.4 \mathrm{~K}$ for TIR). These stations, together with $\mathrm{K}$ and $\mathrm{L}$, all have dry conditions with low vegetation. When there is less vegetation, the influence of soil emissivity on the observed Ka-band brightness temperature becomes larger. Small changes in soil moisture can affect the soil emissivity and will result in biases for MW-LST when a constant emissivity is assumed (as in the current implementation). This points to possible improvements when the scaling to TIR is performed at shorter window lengths, perhaps in 3-month moving windows.

Because MW-LST is scaled directly to TIR-LST, its bias is almost completely determined by the bias between TIR-LST and the site (Table 3 and Fig. 2b). The European FLUXNET sites fall within the MSG domain, and these data years were part of the data on which the scaling of MW-LST is trained (Holmes et al., 2015). Although the mean bias (Fig. 2b) is almost identical, the bias in morning heating ( $\Delta T$, Fig. 2c) has more variation between the two satellite products. It is interesting that generally the satellite products overestimate $\Delta T$ compared to ground data: on average they both overestimate the recorded heating at the stations by about $10 \%$.

\subsection{Cloud tolerance of satellite LST}

To test the stability of the MW-LST for increasing levels of cloudiness, we took a closer look at the four sites in Europe and three in the US (sites A-G). To isolate the effect of clouds on the agreement between satellite and ground observations, we first remove structural differences by fitting a linear regression for each location, based on data with cloudiness below $20 \%$ ( $\Gamma-$ see Sect. $2 \mathrm{c}$ ). We then divide the data into five equal bins of increasing cloud coverage from 0 to $100 \%$. The RMSE and mean difference (bias) between the satellite data and the regression-corrected in situ data is then calculated for each $20 \%$ cloud bin. The purpose is to test the assumption that MW-LST is tolerant to higher levels of cloud coverage.

Figure 3 shows the result of this analysis for locations A$\mathrm{G}$ (from left to right). For each location the data coverage (top row), RMSE (middle row) and bias (bottom row) are displayed for the five $20 \%$ bins of cloudiness. First of all, 

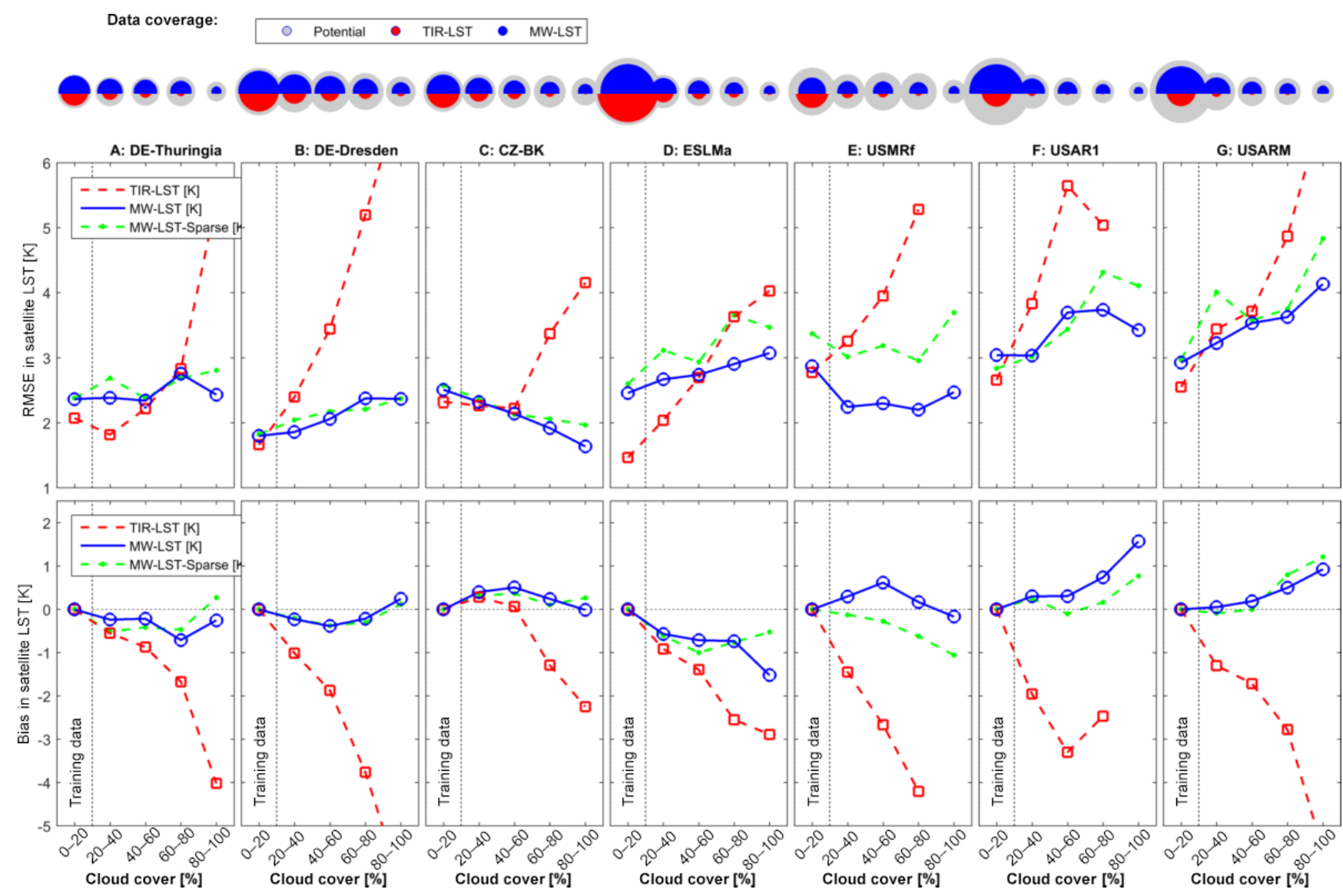

Figure 3. RMSE and bias of satellite LST with regression-corrected in situ data for five levels of cloud cover (( $A_{\text {cloud }}$, Eqs. 8-10). From left to right are locations A-G (see Table 1 for site information). Results for TIR-LST (red) are contrasted with those for MW (blue). Markers indicate that more than 15 days with data were available for a particular cloud cover bin. Green dashed lines indicate the results for MWLST-Sparse. For each site and cloud interval the percentage coverage of the temporal record is depicted in the top row with half-rounds in proportion to the number of data pairs. The potential number of data pairs (grey) refers to the number of in situ data points for each cloud bin. The actual number of data pairs is superimposed on this for MW (blue) and TIR (red).

the large increase in negative bias with increasing cloudiness for the TIR-LST product stands out. At all stations we see a clear negative bias in response to increasing cloudiness for TIR-LST, and the overall agreement between stations is striking. At 40-60\% cloud cover, all stations but one show a significant negative bias for TIR-LST. Above $60 \%$ cloud cover all stations (where TIR-LST is still available, presumably due to failure of the cloud mask) show a negative bias of $2 \mathrm{~K}$ or more. This clearly shows that for TIR-LST we have to accept the hypothesis that clouds affect the satellite LST estimate, even after a cloud mask is applied. It is well known that TIR observations are sensitive to clouds and that a failure to mask for cloud conditions will result in an underestimated LST (for land surface above freezing). Because of this systematic response to clouds, the bias metric by itself is a good indicator of the effect of cloud contamination in clearsky TIR-LST products. The symbols in the top row show the diminishing temporal sampling with increasing cloud cover. When we contrast this with the size of the bias, it is clear that the cloud mask as implemented in the LSA-SAF product (for sites A-D) is not sufficient at removing cloud artifacts. The GOES product (for sites $\mathrm{E}-\mathrm{G}$ ) appears to remove times with high cloud values more completely. Although investigating the efficacy of cloud masks for TIR techniques is not the purpose of this paper, it does help illustrate how cloud effects can be identified with these ground stations.

In clear contrast to the TIR-LST products, the response of MW-LST to increasing cloudiness is much more muted and not as consistent across stations. Stations A, B, C and E show no response in terms of bias, and below $80 \%$ cloud cover there is no station with a MW-LST bias of more than $1 \mathrm{~K}$. One station shows a negative trend (D), and two stations show a positive trend ( $\mathrm{F}$ and $\mathrm{G})$. But only above $80 \%$ cloudiness do these trends result in bias error of greater than $1 \mathrm{~K}$. Because we see both positive and negative biases in the MW-LST analysis, we cannot rely solely on the bias metric to assess the impact of clouds. If there are cancelling biases affecting an individual station, this could suppress the bias. 
Table 4. Validation results by MW satellite (all data, a.m. or p.m. overpass), aggregated for locations A-M (see Table 1).

\begin{tabular}{|c|c|c|c|c|c|c|c|c|}
\hline \multirow[b]{2}{*}{ Satellite } & \multirow[b]{2}{*}{ Overpass } & \multicolumn{3}{|c|}{2009 linear regression } & \multicolumn{3}{|c|}{2015 diurnal scaling } & \multirow[b]{2}{*}{$\mathrm{N}$} \\
\hline & & RMSE & SEE & Bias & RMSE & SEE & Bias & \\
\hline \multirow[t]{3}{*}{ AMSR-E } & & 4.5 & 3 & 0.7 & 3.2 & 2.6 & 0.2 & 6227 \\
\hline & a.m. & 4.4 & 1.9 & 3 & 3.1 & 2.1 & -1.1 & 2817 \\
\hline & p.m. & 4.6 & 2.7 & -1.5 & 3.3 & 2.6 & 1.4 & 3410 \\
\hline \multirow[t]{3}{*}{ AMSR2 } & & 4.4 & 2.4 & 1.6 & 3 & 1.9 & 0.8 & 843 \\
\hline & a.m. & 5 & 1.7 & 3.7 & 2.6 & 2 & -0.7 & 347 \\
\hline & p.m. & 3.8 & 1.6 & -0.3 & 3.2 & 1.6 & 1.9 & 478 \\
\hline \multirow[t]{3}{*}{ WindSat } & & 3.5 & 2.3 & 0.8 & 3.1 & 2.5 & -0.6 & 3080 \\
\hline & a.m. & 3.3 & 2.1 & 1.4 & 3.1 & 2 & -0.2 & 1617 \\
\hline & p.m. & 3.7 & 1.9 & 0.2 & 3 & 2.1 & -0.9 & 1463 \\
\hline \multirow[t]{3}{*}{$\mathrm{SSM} / \mathrm{I}$} & & 3.8 & 2.4 & 0.9 & 3.1 & 2.6 & -0.3 & 5401 \\
\hline & a.m. & 3.5 & 2.3 & 1.1 & 3.1 & 2.2 & 0.2 & 2740 \\
\hline & p.m. & 4 & 2.1 & 0.8 & 3 & 2.3 & -0.8 & 2661 \\
\hline \multicolumn{2}{|c|}{ Average (all sites) } & 4.1 & 2.4 & 1.2 & 3.1 & 2.4 & 0.6 & \\
\hline \multicolumn{2}{|c|}{ Forest (sites: C, E, H) } & 5.1 & 2.1 & 4.3 & 3 & 2 & 0.5 & \\
\hline \multicolumn{2}{|c|}{ Low vegetation } & 3.9 & 2.5 & 0.9 & 3.2 & 2.5 & 0.7 & \\
\hline
\end{tabular}

The increased retrieval error would still be reflected in an increased RMSE. However, the RMSE of MW-LST changes minimally relative to its baseline value at $0-20 \%$ cloudiness and mirrors the size of the bias. This indicates that there is little potential for "hidden" biases behind these numbers. For MW-LST we can therefore reject the hypothesis that clouds affect the satellite LST estimate.

The MW-LST-Sparse product (Eq. 4) adopts the same scaling with TIR as the diurnal MW-LST but has much less sensitivity to the imposed shape of the diurnal model (DTC). For clear skies this distinction is negligible, as apparent from the almost identical values of ubRMSE shown in Table 3. The effect of the clear-sky model is likely to be higher on days with cloudy or partially cloud-covered sky. And although the sparse set only has four-eight observations per day, it allows more samples on days with complex temperature changes. Such days are removed from the MW-DTC product if no good match is found between the diurnal model and observations. We can therefore use the MW-LST-Sparse product to test for undue influence of the DTC model (and its related quality flags) on the relationships between LST errors and cloudiness. The response in bias of MW-LST-Sparse to increasing cloudiness is almost identical to the response of MW-LST for each station (see Fig. 3). In terms of RMSE the sparse set shows values equal to or higher than the diurnallycontinuous MW-LST product, which is not surprising as it does not have the smoothing and quality control associated with the DTC model.

\subsection{All-sky validation by satellite overpass time}

The MW-LST record is a combination of different satellites. In the following analysis the validation results of the MWLST product are broken down by time of day and satellite input record. All data pairs where the minimum temperature at the station stays above freezing are included in this analysis, regardless of cloud cover. It is interesting to compare these results to the much simpler approach that uses a single linear regression model globally (Holmes et al., 2009). Table 4 lists RMSE, SEE and bias for the old and new approach. The statistics are aggregated for all locations as listed in Table 1. The mean scaling with TIR-LST results in a drop in bias for the MW-LST, reducing the average RMSE by $1 \mathrm{~K}$. Part of this reduced RMSE results from the improved characterization of the amplitude of the diurnal cycle, which improves the slope at all times of day and accounts for $0.2 \mathrm{~K}$ of the improvement in RMSE. The impact on the precision (quantified here by SEE) is mixed - on average there is no change. Biggest improvements in all metrics are recorded for the forest locations (sites $\mathrm{C}, \mathrm{E}$ and $\mathrm{H}$ ).

\section{Discussion}

Considering all eight locations used in the cloud analysis, we see little to no response to clouds in terms of bias and RMSE for MW-LST, and this allows us to reject the hypothesis that clouds negatively affect its accuracy. However, for three sites we do find weak and opposing biases at higher cloud coverage which require an explanation. The wavelength of Ka- 
band $(8 \mathrm{~mm})$ is 2 orders of magnitude larger than a typical cloud droplet $(10 \mu \mathrm{m})$. Therefore, any effect of clouds on MW-LST would stem from changes in associated meteorological conditions like atmospheric vapor content and temperature profiles and their potential impact on Ka-band emission processes. According to the zero-order radiative transfer model, an increased atmospheric opacity (through increasing atmospheric water content) increases the weight of the atmospheric contribution to the satellite-measured brightness temperature, relative to the top of vegetation emission. The sign and size of the effect of a change in atmospheric opacity thus depend on the contrast between the atmospheric temperature and the land surface temperature times the effective emissivity. It is therefore possible that this could explain the site-to-site differences in bias as shown in Fig 3. Analyzing the overall effect of the atmosphere on biases in MW-LST will require more detailed atmospheric profile information coupled with a radiative transfer model.

Another possible explanation is that the positive biases recorded at locations $\mathrm{F}$ and $\mathrm{G}$ are related to scale differences between the site and the $0.25^{\circ}$ grid cell. Spatial heterogeneity in LST is likely more pronounced during clear-sky periods when spatially varying soil and vegetation yield a strong influence on the daytime temperature gradients. During cloudy periods the temperature gradients are not as pronounced and more directly linked to the more uniform air temperature. If the mean temperature at the station is generally higher than the areal mean LST, and this bias diminishes with increasing cloudiness, this would be transformed through our clear-sky training into a positive bias for the satellite product at high cloudiness. However, this effect would affect both MW and TIR to the same extent. We have tested this at locations with two stations in contrasting land cover types (A-C). What we found is that indeed it is possible to "rotate" the bias response by changing the weights of the individual stations and that this rotation affects both MW and TIR-LST. This effect of site representation can therefore explain the greater variation in response from station to station for locations where only one station was available (D-G).

\section{Conclusions}

In this paper, a recently developed satellite MW-LST product is compared to ground station data and satellite TIR-based LST products. The MW-LST was developed to complement TIR-LST with a coarser spatial resolution but at a higher temporal resolution. The higher temporal resolution of MW-LST is based on the assumption that MW has a relatively high tolerance to clouds, which allows for observations at times when no TIR observations are possible. This paper tests this assumption by looking at the precision with respect to ground stations for increasing levels of estimated cloudiness. Our analysis is performed at the $0.25^{\circ}$ spatial resolution as predicated by the MW-LST product. At this coarse spatial res- olution, the overall unbiased RMSE between TIR-LST and ground stations during clear-sky days is $2.1 \mathrm{~K}$ for the four locations in the MSG domain, and $2.8 \mathrm{~K}$ for the nine locations in the GOES domain. For the same locations we find that the MW-LST is only slightly higher $(+0.2 \mathrm{~K}$ for both domains).

With increasing cloudiness the RMSE increases significantly for TIR-LST, caused by a matching negative trend in bias that is seen at all seven locations. This demonstrated the known effect that clouds have on TIR estimates of LST. The fact that these trends are so apparent highlights the limitations of current cloud screening techniques as employed in TIR-LST products that are in general use. In clear contrast to this we find a much more limited response in both RMSE and bias for MW-LST. Because of this we conclude that there is no significant direct impact of clouds on the accuracy of the MW-LST product. However, at three stations the size and sign of the response is such that further research is needed to identify the exact causes introducing error in MW-LST. By taking into account the atmospheric humidity and temperature profile, further analysis may investigate the extent to which this mixed response can be explained by atmospheric conditions associated with cloudiness. Alternatively, if a greater database were available of locations with flux tower sites in contrasting land covers, this could be used to isolate the role of scale mismatch between station and the satellite product.

As an immediate outcome the result of this work highlights the utility of MW technology for cloud screening of TIR-LST. This is something that will be explored in future work. Ultimately, the goal is to find the best way of combining MW and TIR technology for the estimation of LST from space.

\section{Data availability}

Time series of MW-LST and TIR-LST covering the locations and time period of this paper are available upon request from the corresponding author. The global source data for MWLST are publicly available. They are aggregated from several data centers, and we would like to thank Goddard Earth Sciences (GES) Data and Information Services Center (DISC) for archiving and distributing TRMM satellite as acquired by NASA's Earth-Sun System Division, the National Snow and Ice Data Center for archiving and distributing Aqua-AMSRE data, and NOAA's Comprehensive Large Array-data Stewardship System (CLASS) for dissemination of Defense Meteorological Satellite Program data. LSA-SAF disseminates EUMETSAT products. This work further used data acquired by the FLUXNET community (fluxnet.ornl.gov) and in particular by the following networks: AmeriFlux and CarboEuropeIP. 
Acknowledgements. This work was funded by NASA through the research grant "The Science of Terra and Aqua" (13-TERAQ130181). We would further like to thank Li Fang (NOAA) for preparation and interpretation of GOES LST.

Edited by: A. Loew

Reviewed by: C. Prigent and one anonymous referee

\section{References}

Aires, F., Prigent, C., Rossow, W. B., and Rothstein, M.: A new neural network approach including first guess for retrieval of atmospheric water vapor, cloud liquid water path, surface temperature, and emissivities over land from satellite microwave observations, J. Geophys. Res.-Atmos., 106, 1488714907, doi:10.1029/2001JD900085, 2001.

Anderson, M. C., Kustas, W. P., Norman, J. M., Hain, C. R., Mecikalski, J. R., Schultz, L., González-Dugo, M. P., Cammalleri, C., d'Urso, G., Pimstein, A., and Gao, F.: Mapping daily evapotranspiration at field to continental scales using geostationary and polar orbiting satellite imagery, Hydrol. Earth Syst. Sci., 15, 223-239, doi:10.5194/hess-15-223-2011, 2011.

André, C., Ottlé, C., Royer, A., and Maignan, F.: Land surface temperature retrieval over circumpolar Arctic using SSM/ISSMIS and MODIS data, Remote Sens. Environ., 162, 1-10, doi:10.1016/j.rse.2015.01.028, 2015.

Baldocchi, D., Falge, E., Gu, L. H., Olson, R., Hollinger, D., Running, S., Anthoni, P., Bernhofer, C., Davis, K., Evans, R., Fuentes, J., Goldstein, A., Katul, G., Law, B., Lee, X. H., Malhi, Y., Meyers, T., Munger, W., Oechel, W., U, K., Pilegaard, K., Schmid, H. P., Valentini, R., Verma, S., Vesala, T., Wilson, K., and Wofsy, S.: FLUXNET: A new tool to study the temporal and spatial variability of ecosystem-scale carbon dioxide, water vapor, and energy flux densities, B. Am Meteorol. Soc., 82, 24152434, 2001.

Catherinot, J., Prigent, C., Maurer, R., Papa, F., Jiménez, C., Aires, F., and Rossow, W. B.: Evaluation of "all weather" microwave-derived land surface temperatures with in situ CEOP measurements, J. Geophys. Res.-Atmos., 116, D23105, doi:10.1029/2011JD016439, 2011.

Ermida, S. L., Trigo, I. F., DaCamara, C. C., Göttsche, F. M., Olesen, F. S., and Hulley, G.: Validation of remotely sensed surface temperature over an oak woodland landscape-The problem of viewing and illumination geometries, Remote Sens. Environ., 148, 16-27, 2014.

Friedl, M. A., Sulla-Menashe, D., Tan, B., Schneider, A., Ramankutty, N., Sibley, A., and Huang, X.: MODIS Collection 5 global land cover: Algorithm refinements and characterization of new datasets, Remote Sens. Environ., 114, 168-182, 2010.

Göttsche, F.-M., and Olesen, F. S.: Modelling of diurnal cycles of brightness temperature extracted from METEOSAT data, Remote Sens. Environ., 76, 337-348, doi:10.1016/S00344257(00)00214-5, 2001.

Göttsche, F.-M., Olesen, F.-S., and Bork-Unkelbach, A.: Validation of land surface temperature derived from MSG/SEVIRI with in situ measurements at Gobabeb, Namibia, Int. J. Remote Sens., 34, 3069-3083, doi:10.1080/01431161.2012.716539, 2013.
Göttsche, F.-M., Olesen, F.-S., Trigo, I. F., Bork-Unkelbach, A. and Martin, M. A.: Long Term Validation of Land Surface Temperature Retrieved from MSG/SEVIRI with Continuous in-Situ Measurements in Africa, Remote Sens., 8, 410, doi:10.3390/rs8050410, 2016.

Holmes, T. R. H., De Jeu, R. A. M., Owe, M., and Dolman, A. J.: Land surface temperature from $\mathrm{Ka}$ band $(37 \mathrm{GHz})$ passive microwave observations, J. Geophys. Res.-Atmos., 114, D04113, doi:10.1029/2008JD010257, 2009.

Holmes, T. R. H., Jackson, T. J., Reichle, R. H., and Basara, J. B.: An assessment of surface soil temperature products from numerical weather prediction models using ground-based measurements, Water Resour. Res., 48, W02531, doi:10.1029/2011WR010538, 2012.

Holmes, T. R. H., Crow, W. T., Yilmaz, M. T., Jackson, T. J., and Basara, J. B.: Enhancing model-based land surface temperature estimates using multiplatform microwave observations, J. Geophys. Res.-Atmos., 118, 577-591, doi:10.1002/jgrd.50113, $2013 a$.

Holmes, T. R. H., Crow, W. T., and Hain, C.: Spatial patterns in timing of the diurnal temperature cycle, Hydrol. Earth Syst. Sci., 17, 3695-3706, doi:10.5194/hess-17-3695-2013, 2013b.

Holmes, T. R. H., Crow, W. T., Hain, C. R., Anderson, M., and Kustas, W. P.: Diurnal temperature cycle as observed by thermal infrared and microwave radiometers, Remote Sens. Environ., 158C, 110-125, doi:10.1016/j.rse.2014.10.031, 2015.

Jackson, T. J., Le Vine, D. M., Hsu, A. Y., Oldack, A., Starks, P. J., Swift, C. T., Isham, J. D., and Haken, M.: Soil moisture Mapping at regional scales using microwave radiometry: The Southern Great Plains hydrology experiment, IEEE Trans. Geosci. Remote Sens., 37, 2136-2149, 1999.

Lei, F., Crow, W. T., Shen, H., Parinussa, R. M., and Holmes, T. R. H.: The Impact of Local Acquisition Time on the Accuracy of Microwave Surface Soil Moisture Retrievals over the Contiguous United States, Remote Sens., 7, 13448-13465, doi:10.3390/rs71013448, 2015.

Marek, M. V., Janouš, D., Taufarová, K., Havránková, K., Pavelka, M., Kaplan, V., and Marková, I.: Carbon exchange between ecosystems and atmosphere in the Czech Republic is affected by climate factors, Environ. Pollut., 159, 1035-1039, doi:10.1016/j.envpol.2010.11.025, 2011.

Norman, J. M. and Becker, F.: Terminology in thermal infrared remote sensing of natural surfaces, Agr. Forest Meteorol., 77, 153166, doi:10.1016/0168-1923(95)02259-Z, 1995.

Owe, M. and Van de Griend, A. A.: On the relationship between thermodynamic surface temperature and high-frequency $(37 \mathrm{GHz})$ vertically polarized brightness temperature under semiarid conditions, Int. J. Remote Sens., 22, 3521-3532, doi:10.1080/01431160110063788, 2001.

Owe, M., De Jeu, R. A. M., and Holmes, T. R. H.: Multisensor historical climatology of satellite-derived global land surface moisture, J. Geophys. Res.-Earth, 113, F01002, doi:10.1029/2007JF000769, 2008.

Parinussa, R. M., Holmes, T. R. H., Yilmaz, M. T., and Crow, W. T.: The impact of land surface temperature on soil moisture anomaly detection from passive microwave observations, Hydrol. Earth Syst. Sci., 15, 3135-3151, doi:10.5194/hess-15-31352011, 2011. 
Prigent, C., Jimenez, C., and Aires, F.: Towards "all weather", long record, and real-time land surface temperature retrievals from microwave satellite observations, J. Geophys. Res.-Atmos., 121, 5699-5717, doi:10.1002/2015JD024402, 2016.
Van Wijk, W. R. and Ubing, D. S.: Radiation, in Physics of plant environment., North-Holland Publ. Co., Amsterdam, 62-101, 1963. 